\title{
Diaphragm Activation via High Frequency Spinal Cord Stimulation in a Rodent Model of Spinal Cord Injury
}

\author{
Krzysztof E. Kowalski, Ph.Da, Yee-Hsee Hsieh, Ph.D. ${ }^{b}$, Thomas E Dick, Ph.D. ${ }^{b}$, and Anthony \\ F. DiMarco, M.D. ${ }^{\mathrm{C}}$ \\ Krzysztof E. Kowalski: kek5@case.edu; Yee-Hsee Hsieh: yee-hsee.hsieh@case.edu; Thomas E Dick: \\ thomas.dick@case.edu; Anthony F. DiMarco: afd3@case.edu \\ aDepartment of Medicine, Case Western Reserve University and MetroHealth Medical Center, \\ 2500 MetroHealth Drive, R550, Cleveland, OH, 44109, USA \\ bDivision of Pulmonary and Critical Care Medicine, Department of Medicine, Case Western \\ Reserve University, Biomedical Research Bldg. BRB B55, 10900 Euclid Avenue, Cleveland, OH \\ 44106, USA \\ 'Department of Physical Medicine \& Rehabilitation, Case Western Reserve University and \\ MetroHealth Medical Center, 2500 MetroHealth Drive, R551, Cleveland, OH, 44109, USA
}

\section{Abstract}

As demonstrated in a canine model, high frequency spinal cord stimulation (HF-SCS) is a novel and more physiologic method of electrical activation of the inspiratory muscles compared to current techniques. The dog model, however, has significant limitations due to cost and societal concerns. Since the rodent respiratory system is also a relevant model for study of neuronal circuitry function, the aims of the present study were to a) assess the effects of HF-SCS and b) determine the methodology of application of this technique in rats. In 9 Sprague Dawley rats, diaphragm multiunit and single motor unit EMG activity was assessed during spontaneous breathing and HF-SCS applied on the ventral epidural surface of the spinal cord at the T2 level following $\mathrm{C} 1$ spinal section. As in dogs, HF-SCS results in the activation of the diaphragm at physiological firing frequencies and the generation of large inspired volumes. Mean maximum firing frequencies of the diaphragm during spontaneous breathing and HF-SCS were $23.3 \pm 1.4 \mathrm{~Hz}$ (range: $9.8-51.6 \mathrm{~Hz}$ ) and $26.6 \pm 1.3 \mathrm{~Hz}$; range: $12.0-72.9 \mathrm{~Hz}$, respectively, at comparable inspired volumes. Moreover, HF-SCS was successful in pacing these animals over a 60-min period without evidence of system fatigue. Our results suggest that, similar to the dog model, HFSCS in the rat results in activation of spinal cord tracts which synapse with the phrenic motoneuron pool, allowing processing of the stimulus and consequent physiologic activation of the inspiratory muscles. The rat may be a useful model for further studies evaluating phrenic motoneuron physiology.

(c) 2013 Elsevier Inc. All rights reserved.

Corresponding Author: Anthony F. DiMarco, MD, Department of Physiology and Biophysics, Case Western Reserve University, MetroHealth Medical Center, Rammelkamp Center for Education \& Research, 2500 MetroHealth Drive, R551, Cleveland, OH 44109-1998, Phone: 216-778-3906, Fax: 216-778-8589, afd3@ case.edu.

Publisher's Disclaimer: This is a PDF file of an unedited manuscript that has been accepted for publication. As a service to our customers we are providing this early version of the manuscript. The manuscript will undergo copyediting, typesetting, and review of the resulting proof before it is published in its final citable form. Please note that during the production process errors may be discovered which could affect the content, and all legal disclaimers that apply to the journal pertain. 


\section{Keywords}

spinal cord stimulation; inspiratory muscles; spinal cord injury

\section{Introduction}

In our previous investigations using a canine model (DiMarco and Kowalski, 2009, 2010, 2011), we demonstrated that high frequency spinal cord stimulation (HF-SCS) applied in the region of the upper thoracic spinal cord results in activation of the inspiratory muscles in a more physiologic fashion (DiMarco and Kowalski, 2009, 2010, 2011). In contrast to direct electrical stimulation of motor roots and nerves (Glenn, 1980; Glenn et al., 1980; DiMarco et al., 1994, 2002, 2005a, 2005b, 2006b), this novel method evokes an asynchronous pattern of inspiratory activity (DiMarco and Kowalski, 2009, 2010, 2011). Additionally, single motor units (SMU) are recruited to fire diaphragmatic action potentials at discharge frequencies in the physiologic range (DiMarco and Kowalski, 2009), rather than at the frequency of the stimulus. In fact, SMU firing frequency rates are not significantly different than those occurring during spontaneous breathing at comparable inspired volumes (DiMarco and Kowalski, 2009, 2010, 2011).

Since the canine spinal cord is similar in size to humans and the central organization of respiratory neurons and spinal pathways is also comparable, this species has provided a very useful model for translational research with regard to the evaluation of electrical stimulation techniques to restore respiratory muscle function (DiMarco et al., 2002, 2005a, 2005b, 2006a, 2006b, 2009). The canine species, however, is increasingly less accessible compared to animals lower on the phylogenic scale, such as rodents, due to expense and societal concerns (Courtine, et al., 2007; Kastner and Gauthier, 2008). Since it appears that the brainstem and spinal organization of the respiratory neuronal structures is preserved phylogenetically, rodents may be a more practical species to evaluate the neuronal circuitry that controls respiratory muscle activity. More specifically, the rodent may be an ideal model to investigate the consequences of spinal cord injury, neuronal plasticity, the effects of potential repair strategies and other respiratory physiological assessments (de Almeida, et al., 2010; Dougherty et al., 2012; Kastner and Gauthier, 2008; Lane et al., 2008, 2009, 2012; Seven et al., 2012).

The purpose of the present investigation, therefore, was to evaluate the effects of HF-SCS on diaphragm activation in a rodent model of spinal cord injury. If the results of HF-SCS in spinal-transected rats are similar to those in dogs, then not only would we demonstrate that HF-SCS is effective in physiologically activating the inspiratory muscles in a separate mammalian species, but also provide the initial foundation and basic methodology of this new stimulation technique in a more advantageous species. Ultimately, HF-SCS may provide a useful tool for additional physiological studies involving respiratory motoneuron function both in health and disease.

\section{Material and Methods}

Experiments were performed in adult, male Sprague Dawley rats $(n=9$, body weight $433 \pm$ $4 \mathrm{~g}$ ) with the approval of the Institutional Animal Care and Use Committee of Case Western Reserve University. Rats were anesthetized with Urethane ( $1 \mathrm{~g} / \mathrm{kg}$, intraperitoneal bolus injection) and supplemental anesthesia $(0.1 \mathrm{~g} / \mathrm{kg}$ intravenous) was provided as needed. A femoral vein and artery were cannulated to administer supplemental fluids and to monitor arterial blood pressure (Validyne Engineering, Northridge, CA). An endotracheal tube was sutured into the trachea just below the larynx. Body temperature was maintained between 
$37-38^{\circ} \mathrm{C}$ using a homeothermic blanket (Harvard Apparatus, Cambridge, MA). End-tidal $\mathrm{PCO}_{2}$ was monitored with a rapidly responding $\mathrm{CO}_{2}$ analyzer (CapStar- $100 \mathrm{CO}_{2}$ Analyzer, CWE Inc., Ardmore, PA). Tidal volume was recorded by electrical integration of the flow signal from a pneumotachometer (Series 8420, Hans Rudolph Inc., Kansas City, MO), which was connected directly to the tracheostomy tube. The accuracy of the displayed volume values were verified by calibration with an adjustable calibration syringe (volume range between 1-20 ml). In each animal, following measurement of spontaneous activity, the dura mater was opened, and the spinal cord was sectioned at the high $\mathrm{C} 1$ level and subsequently at the $\mathrm{C} 8$ level, using watchmaker forceps under microscopic control. Complete section was verified by lifting a hook across the area of transection and also visually post-mortem.

\section{Diaphragmatic EMG recordings}

Bipolar teflon-coated, silver wire (0.010" bare, 0.013" coated) (A-M Systems, Carlsborg, WA) un-insulated at their terminal $\sim 2 \mathrm{~mm}$ and $<1 \mathrm{~mm}$ were used to assess multi-unit and single motor unit electromyographic (EMG) recordings of the costal diaphragm, respectively. Diaphragmatic EMG potentials were amplified (1000-10,000 times) and filtered (0.05-5.0 kHz) (Grass Technologies P511 Amplifier, AstroMed, Inc., West Warwick, RI). All recordings were monitored and stored for offline analysis on a computer utilizing a data acquisition and analyzing system (Spike2 with 1401 interface, Cambridge Electronic Design, Cambridge, UK).

\section{Spinal cord stimulation (SCS)}

A laminectomy was performed at the T1-T3 level of the vertebral column. Teflon-coated silver wire (diameter: 0.010" bare, 0.013" coated, A-M Systems) was used as the stimulating electrode by uninsulating $\sim 1 \mathrm{~mm}$ at the tip. This electrode was placed on the ventral epidural surface of the spinal cord under direct vision at the T3 level and advanced more rostrally until maximal inspired volume generation was achieved (T2 level). In each animal, the location of the tip of the stimulating electrode was verified post-mortem. A square-wave pulse stimulator (Model S44, Grass Technologies) equipped with a stimulus isolation unit (PSIU6, Grass Technologies) provided the electrical stimulus pulses (100-500 $\mathrm{Hz}, 0-1.5 \mathrm{~mA}, 0.2 \mathrm{~ms}$ ). Our evaluation was limited to $1.5 \mathrm{~mA}$ since higher levels of stimulation resulted in jerking movements of the trunk muscles. Three protocols were performed to evaluate HF-SCS in rodents.

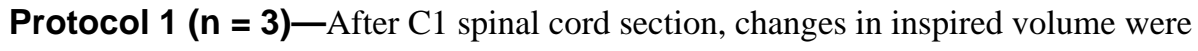
measured during SCS over a wide range of stimulus currents $(0.2-1.5 \mathrm{~mA})$ and stimulus frequencies $(100-500 \mathrm{~Hz})$ at a fixed pulse width of $0.2 \mathrm{~ms}$. Of note, our evaluation was limited to a maximum current of $1.5 \mathrm{~mA}$ since higher levels of stimulus current evoked body movement and, therefore, do not have a practical application. In each animal, the inspiratory capacity was taken as the volume required to inflate the lungs from FRC to TLC, defined as an airway pressure of $+30 \mathrm{cmH}_{2} \mathrm{O}$ according to standard lung function measurements (Crémillieux et al., 2008; Mink et al., 2004).

Protocol $2(n=6)$-Diaphragmatic EMG recording electrodes were placed in the body of the costal diaphragm to assess motor unit activity during spontaneous breathing and HF-SCS $(300 \mathrm{~Hz}, 0.5-0.6 \mathrm{~mA}, 0.2 \mathrm{~ms})$ at the $\mathrm{T} 2$ spinal level following complete $\mathrm{C} 1$ spinal section. Stimulus amplitude was adjusted to approximate the magnitude of inspired volume observed during spontaneous breathing. Five to 10 recording sites were sampled in each rat for SMU recordings. At each site, 1-4 SMUs were distinguished on the basis of their distinct morphology. Several breaths were analyzed at each recording site. 
Protocol $3(\mathbf{n}=\mathbf{3})$-The capacity of HF-SCS to maintain artificial ventilation was assessed by repetitively applying a fixed stimulus paradigm over $60 \mathrm{~min}$. The stimulus parameters $(300 \mathrm{~Hz}, 0.5-0.6 \mathrm{~mA}, 0.2 \mathrm{~ms})$, which resulted in inspired volumes observed during spontaneous breathing were applied at a stimulus train rate (mean; $65 \pm 2$ breaths/ min; range: $63-70$ breaths $/ \mathrm{min}$ ) that maintained end-tidal $\mathrm{PCO}_{2}$ between $35-40 \mathrm{mmHg}$. Mean inspiratory time was $0.33 \pm 0.01 \mathrm{~s}$. During sustained pacing, inspired volume, endtidal $\mathrm{PCO}_{2}$, oxygen saturation, mean blood pressure and heart rate were continuously monitored.

\section{Data Analysis}

Single motor unit analyses included determination of mean peak discharge frequencies assessed over several consecutive breaths. The discharge frequency of each SMU during spontaneous breathing and during HF-SCS was plotted against the number of motor units recorded. Comparisons were made, where applicable, using repeated-measures ANOVA and post hoc Newman-Keuls tests. A p value $<0.05$ was considered statistically significant. All data are reported as means $\pm \mathrm{SE}$.

\section{Results \\ Protocol 1}

The effects of HF-SCS on inspired volume generation during stimulation at 100, 300 and $500 \mathrm{~Hz}$ over a range of stimulus currents in a representative animal are shown in Fig. 1. At each stimulus current, inspired volume generation was greater at 300 compared to $100 \mathrm{~Hz}$. At $500 \mathrm{~Hz}$, inspired volume generation was somewhat less compared to $300 \mathrm{~Hz}$ at each stimulus current.

Mean inspired volumes during SCS over a wide range of stimulus frequencies $(100-500 \mathrm{~Hz})$ and amplitudes (0.2-1.5 mA) are provided in Fig. 2. For reference, mean tidal volume observed during spontaneous breathing $(1.66 \pm 0.03 \mathrm{ml})$ and mean inspiratory capacity $(12.57 \pm 0.29 \mathrm{ml})$ are indicated by the dotted lines. With HF-SCS at each stimulus frequency, the magnitude of inspired volume increased progressively with increasing stimulus current until a plateau was reached at $\sim 0.8 \mathrm{~mA}$. Moreover, at stimulus currents of $0.5 \mathrm{~mA}$ and higher, inspired volume generation was significantly higher at 200 and $300 \mathrm{~Hz}$ than at $100 \mathrm{~Hz}$ ( $\mathrm{p}<0.05$ for each comparison). HF-SCS at higher stimulus frequencies (400 and $500 \mathrm{~Hz}$ ) resulted in significantly lower inspired volumes than that achieved with $300 \mathrm{~Hz}$ ( $\mathrm{p}<0.05$ for each comparison). For example, HF-SCS ( $1 \mathrm{~mA}, 0.2 \mathrm{~ms}$ ) at stimulus frequencies of 100, 300, 400 and $500 \mathrm{~Hz}$ evoked mean inspired volumes of $3.0 \pm 0.7,5.0 \pm$ $0.5,3.9 \pm 0.9$ and $3.6 \pm 0.9$ respectively. In summary, at any given stimulus current, mean inspired volumes during HF-SCS were significantly higher at $300 \mathrm{~Hz}$ compared to those achieved with stimulation at 100, 400 and $500 \mathrm{~Hz}(\mathrm{p}<0.05)$.

\section{Protocol 2}

The diaphragmatic EMG patterns during spontaneous breathing (left panel) and during HFSCS (300 Hz, 0.5-0.6 mA, $0.2 \mathrm{~ms})$ (right panel) at similar inspired volumes are shown for one animal in Fig. 3. Similar to spontaneous breathing, HF-SCS resulted in an asynchronous EMG pattern. Similar patterns of diaphragm EMG activity were observed in each animal.

Representative examples of SMU recordings from the diaphragm during spontaneous breathing and HF-SCS are provided in Fig. 4. Raw EMGs are displayed above a plot of instantaneous firing frequencies of each SMU. Superimposition of the SMUs (at the right of each EMG) confirmed their similar morphology. In this instance, peak firing frequencies were 19.5 and $12.6 \mathrm{~Hz}$ during spontaneous breathing and HF-SCS, respectively. 
We recorded 68 and 50 SMUs during spontaneous breathing and HF-SCS, respectively. Histograms of the discharge frequencies of all recorded SMUs are shown in Fig. 5. Importantly, these measurements were made during comparable mean inspired volumes: 3.2 $\pm 0.1 \mathrm{ml}$ for spontaneous breathing and $3.3 \pm 0.1 \mathrm{ml}$ for HF-SCS. There was a similar broad range of firing frequencies during spontaneous breathing $(12.0-72.9 \mathrm{~Hz})$ and during HFSCS $(9.8-51.6 \mathrm{~Hz})$. Consequently, mean firing frequencies during spontaneous breathing $(26.6 \pm 1.3 \mathrm{~Hz})$ and HF-SCS $(23.3 \pm 1.4 \mathrm{~Hz})$ were not significantly different.

\section{Protocol 3}

The mean effects of continuous HF-SCS pacing applied over a 60-min period on inspired volume, end-tidal $\mathrm{PCO}_{2}$, oxygen saturation, blood pressure and pulse rate are shown in Fig. 6 and Table I. The stimulus parameters were set at the same values $(300 \mathrm{~Hz}, 0.6 \mathrm{~mA}, 0.2 \mathrm{~ms}$, 65 breaths $/ \mathrm{min}$ ) in each animal. Throughout the period of stimulation, inspired volume generation remained unchanged. Mean inspired volume was $3.3 \pm 0.1$ initially and $3.1 \pm 0.1$ $\mathrm{ml}$ at the end of the hour and did not change significantly over the period of stimulation. In addition, mean blood pressure and heart rate remained stable throughout the period of stimulation $\left(97 \pm 7-101 \pm 9 \mathrm{mmHg}\right.$ and $\left.225 \pm 16-234 \pm 23 \mathrm{~min}^{-1}\right)$. There was no observable limb motion during continuous HF-SCS at these stimulus intensities.

Section of the spinal cord below the level of the phrenic motoneuron pools (C6 spinal level) abolished all diaphragm EMG activity.

\section{Discussion}

This is the first study to demonstrate that HF-SCS using a single electrode positioned on the ventral surface of the upper thoracic spinal cord results in activation of the phrenic motoneurons via spinal cord pathways in a rodent model. As with the canine model, phrenic motoneuron activation is evidenced by the asynchronous pattern of diaphragmatic EMG which resembled that occurring during spontaneous breathing, and the SMU firing frequencies were not significantly different than those observed during spontaneous breathing. In contrast to conventional electrical stimulation techniques, HF-SCS allows for processing of the stimulus within the motoneuron pools resulting in a more physiological recruitment pattern. This method stands in contrast to direct phrenic nerve stimulation, which results in synchronous activation of all axons within the electric field and the development of a single action potential following each stimulus (Glenn, 1980; Glenn et al., 1980; DiMarco et al., 1987, 1994, 2005b).

The specific spinal cord pathways and neural substrate mediating phrenic motoneuron activation during HF-SCS are not known. In the dog model, however, we determined that tracts located bilaterally in the ventro-lateral portion of the spinal cord (DiMarco and Kowalski, 2012 in press) mediated the observed responses. Interestingly, Decima and colleagues $(1697,1969 \mathrm{a}, 1969 \mathrm{~b})$ described afferents from the lower thoracic and abdominal muscles (T9-T13) in cats, which are located in the ventro-lateral portion of the spinal cord, that reflexively facilitate phrenic motoneuron activity via spinal cord pathways (intercostal to phrenic reflex). It is possible that activation of the phrenic motoneurons via HF-SCS may be the result of stimulation of these same excitatory tracts. Moreover, the results of the present study suggest that similar tracts may also be present in rodents.

\section{Comparison to Previous Studies}

Obviously, tidal volume and respiratory rate are significantly different between rodents and dogs (mean 3.2 vs. $290 \mathrm{ml}$ and mean 78 vs. 7 breaths $/ \mathrm{min}$ ). Therefore, it is not surprising that the mean peak firing frequencies during spontaneous and HF-SCS breathing are also 
different. Based upon our previous work, mean peak firing frequency recorded from the diaphragm in dogs during spontaneous and HF-SCS breathing when matched for inspired volume were $10.8 \pm 0.3$ and $10.4 \pm 0.3 \mathrm{~Hz}$ (NS), respectively (DiMarco and Kowalski, 2009). This compares to a mean $26.6 \pm 1.3 \mathrm{~Hz}$ and $23.2 \pm 1.4 \mathrm{~Hz}$ (NS) during spontaneous and HF-SCS breathing in rodents, as demonstrated in the present study. The fact that mean peak firing frequencies during HF-SCS were not significantly different compared to spontaneous breathing in both species lends further support to the concept that the HF-SCS method results in activation of phrenic motoneurons via spinal networks.

Motoneuron firing properties were previously investigated by Kong and Berger (1986) by recording from single phrenic motoneuron axons isolated from the phrenic nerve trunk. Mean discharge frequencies ranged between 34 and 76 spikes/s (mean 57 and 55 spikes for early- and late-onset types, respectively). Similar responses were observed in a cat model (St John and Bartlett, 1979). While the specific cause of these higher values of motoneuron firing frequencies compared to those reported in the present study is not clear, the difference may relate to the surgical preparation of the rodents. The rodents in these previous studies were subjected to bilateral vagotomy and phrenicotomy. Use of different anesthetic agents may have also impacted the pattern of discharge frequencies.

\section{Study Limitations}

Normal breathing is accompanied by a specific pattern of motor unit recruitment according to Henneman's Size Principle, which is based upon motoneuron size (Henneman et al., 1965). While it is probable that HF-SCS results in this physiologic recruitment pattern, specific studies to address this issue have not yet been performed.

In our previous studies in canines, we determined that HF-SCS resulted in coincident inspiratory intercostal muscle activation via spinal cord pathways in addition to phrenic activation (DiMarco and Kowalski, 2009, 2010, 2011). While it is probable that the intercostal muscles were also activated in the rodents, this was not investigated in the present study.

While the present studies were performed following spinal cord section at the $\mathrm{C} 1$ level, it is possible that transected axons from bulbo- and proprio- spinal interneurons remained functional (McDonald, 1972). Therefore, a potential mechanism by which the inspiratory motoneuronal pools were activated via HF-SCS is electrical stimulation of these severed tracts. A different experimental design involving prolonged study duration of several days to allow degeneration of these axons following spinal cord section would be necessary to evaluate this possibility.

\section{Potential Investigational Applications}

Due to the apparent phylogenetic preservation of respiratory central nervous system networks, the rat has provided a useful experimental model in which respiratory motor control can be assessed (Courtine et al., 2007; Kastner and Gauthier, 2008). Moreover, the effects of a variety of injury types, post injury mechanisms of neuronal plasticity, and the impact of specific repair strategies have also been evaluated (de Almeida et al., 2010; Dougherty et al., 2012; Kastner and Gauthier, 2008; Lane et al., 2008, 2009, 2012; Seven et al., 2012). In this regard, HF-SCS may provide an additional tool to evaluate phrenic motoneuron function. For example, by applying HF-SCS before and after a given intervention, the impact of that maneuver on the integrity of phrenic motoneuron function can be assessed. 


\section{Conclusion}

In summary, HF-SCS in rodents results in similar activation of the phrenic motoneuron pools via spinal cord pathways as that described previously in canine studies. It should be noted, however, that the single motor unit firing frequencies of diaphragm motor units is significantly higher in rats compared to dogs. Additionally, the methodology of HF-SCS in rodents is also described. Just as diaphragm function can be evaluated using phrenic nerve stimulation with a fixed and reproducible level of electrical stimulation, so too can phrenic motoneuron function be evaluated utilizing HF-SCS. For example, the effects of a variety of chemicals including hypoxemia, hypercapnia or other exogenous agents, physical stimuli including trauma, various restorative strategies, and plasticity on phrenic motoneuron function can be assessed directly.

\section{Acknowledgments}

The authors wish to thank Ms. Dana Hromyak for her technical expertise in the preparation of this manuscript.

\section{Abbreviations}

HF-SCS high frequency spinal cord stimulation

EMG electromyogram

SMU single motor unit

SCS spinal cord stimulation

\section{References}

Courtine G, Bunge MB, Fawcett JW, Grossman RG, Kaas JH, Lemon R, Maier I, Martin J, Nudo RJ, Ramon-Cueto A, Rouiller EM, Schnell L, Wannier T, Schwab ME, Edgerton VR. Can experiments in nonhuman primates expedite the translation of treatments for spinal cord injury in humans? Nat. Med. 2007; 13:561-566. [PubMed: 17479102]

Crémillieux Y, Servais S, Berthezène Y, Dupuich D, Boussouar A, Stupar V, Pequignot J. Effects of ozone exposure in rat lungs investigated with hyperpolarized ${ }^{3} \mathrm{He}$ MRI. J Magn. Reson. Imaging. 2008; 27:771-776. [PubMed: 18383246]

de Almeida AT, Al-Izki S, Denton ME, Kirkwood PA. Patterns of expiratory and inspiratory activation for thoracic motoneurones in the anaesthetized and the decerebrate rat. J. Physiol. 2010; 588:27072729. [PubMed: 20530111]

Decima EE, von Euler C, Thoden U. Spinal intercostal-phrenic reflexes. Nature. 1967; 214:312-313. [PubMed: 5231342]

Decima EE, von Euler C. Excitability of phrenic motoneurones to afferent input from lower intercostal nerves in the spinal cat. Acta. Physiol. Scand. 1969a; 75:580-591. [PubMed: 5261232]

Decima EE, von Euler C, Thoden U. Intercostal-to-phrenic reflexes in the spinal cat. Acta. Physiol. Scand. 1969b; 75:568-579. [PubMed: 4243231]

DiMarco AF, Altose MD, Cropp A, Durand D. Activation of the inspiratory intercostal muscles by electrical stimulation of the spinal cord. Am. Rev. Respir. Dis. 1987; 136:1385-1390. [PubMed: 3688644]

DiMarco AF, Supinski GS, Petro JA, Takaoka Y. Evaluation of intercostal muscle pacing to provide artificial ventilation in quadriplegics. Am. J. Resir. Crit. Care Med. 1994; 150:934-940.

DiMarco AF, Onders RP, Kowalski KE, Miller ME, Ferek S, Mortimer JT. Phrenic nerve pacing in a tetraplegic patient via intramuscular diaphragm electrodes. Am. J. Respir. Crit. Care Med. 2002; 166:1604-1606. [PubMed: 12471076]

DiMarco AF, Onders RP, Ignagni A, Kowalski KE, Mortimer JT. Phrenic nerve pacing via intramuscular diaphragm electrodes in tetraplegic subjects. Chest. 2005a; 127:671-678. [PubMed: 15706014] 
DiMarco AF, Takaoka Y, Kowalski KE. Combined intercostal and diaphragm pacing to provide artificial ventilation in patients with tetraplegia. Arch. Phys. Med. Rehabil. 2005b; 86:1200-1207. [PubMed: 15954060]

DiMarco AF, Kowalski KE, Geertman RT, Hromyak DR. Spinal cord stimulation: a new method to produce cough in patients with spinal cord injury. Am. J. Respir. Crit. Care Med. 2006a; 173:1386-1389. [PubMed: 16543552]

DiMarco AF, Onders RP, Ignagni A, Kowalski KE. Inspiratory muscle pacing in spinal cord injury: case report and clinical commentary. J. Spinal Cord Med. 2006b; 29:95-108. [PubMed: 16739553]

DiMarco AF, Kowalski KE, Geertman RT, Hromyak DR. Lower thoracic spinal cord stimulation to restore cough in patients with spinal cord injury: results of a National Institutes of Healthsponsored clinical trial. Part I: methodology and effectiveness of expiratory muscle activation. Arch. Phys. Med. Rehabil. 2009; 90:717-725. [PubMed: 19406289]

DiMarco AF, Kowalski KE. High frequency spinal cord stimulation of inspiratory muscles in dogs: a new method of inspiratory muscle pacing. J. Appl. Physiol. 2009; 107:662-669. [PubMed: 19520839]

DiMarco AF, Kowalski KE. Intercostal muscle pacing with high frequency spinal cord stimulation in dogs. Respir. Physiol. Neurobiol. 2010; 171:218-224. [PubMed: 20338266]

DiMarco AF, Kowalski KE. Distribution of electrical activation to the external intercostal muscles during high frequency spinal cord stimulation in dogs. J. Physiol. 2011; 589:1383-1395. [PubMed: 21242258]

DiMarco AF, Kowalski KE. Spinal pathways mediating phrenic activation during high frequency spinal cord stimulation. Respir. Physiol. Neurobiol. 2012 in press.

Dougherty BJ, Lee KZ, Lane MA, Reier PJ, Fuller DD. Contribution of the spontaneous crossedphrenic phenomenon to inspiratory tidal volume in spontaneously breathing rats. J. Appl. Physiol. 2012; 112:96-105. [PubMed: 22033536]

Glenn WW. The treatment of respiratory paralysis by diaphragm pacing. Ann. Thorac. Surg. 1980; 30:106-109. [PubMed: 6968183]

Glenn WW, Hogan JF, Phelps ML. Ventilatory support of the quadriplegic patient with respiratory paralysis by diaphragm pacing. Surg. Clin. North Am. 1980; 60:1055-1078. [PubMed: 6968983]

Henneman E, Somjen G, Carpenter DO. Functional significance of cell size in spinal motoneurons. J. Neurophysiol. 1965; 28:560-580. [PubMed: 14328454]

Kastner A, Gauthier P. Are rodents an appropriate pre-clinical model for treating spinal cord injury? Examples from the respiratory system. Exp. Neurol. 2008; 213:249-256. [PubMed: 18675802]

Kong FJ, Berger AJ. Firing properties and hypercapnic responses of single phrenic motor axons in the rat. J. Appl. Physiol. 1986; 61:1999-2004. [PubMed: 3027021]

Lane MA, Fuller DD, White TE, Reier PJ. Respiratory neuroplasticity and cervical spinal cord injury: translational perspectives. Trends Neurosci. 2008; 31:538-547. [PubMed: 18775573]

Lane MA, Lee KZ, Fuller DD, Reier PJ. Spinal circuitry and respiratory recovery following spinal cord injury. Respir. Physiol. Neurobiol. 2009; 169:123-132. [PubMed: 19698805]

Lane MA, Lee KZ, Salazar K, O'Steen BE, Bloom DC, Fuller DD, Reier PJ. Respiratory function following bilateral mid-cervical contusion injury in the adult rat. Exp. Neurol. 2012; 235:197-210. [PubMed: 21963673]

McDonald WI. The time course of conduction failure during degeneration of a central tract. Exp. Brain Res. 1972; 14:550-556. [PubMed: 4340073]

Mink SN, Gonzalez X, Duke K, Bautista E, Tan L. Lung volume reduction surgery in canine model of predominantly upper lobe emphysema. Chest. 2004; 125:633-643. [PubMed: 14769748]

Seven YB, Mantilla CB, Zhan WZ, Sieck GC. Non-stationarity and power spectral shifts in EMG activity reflect motor unit recruitment in rat diaphragm muscle. Respir. Physiol. Neurobiol. 2012 [Epub ahead of print].

St. John WM, Bartlette D Jr. Comparison of phrenic motoneuron responses to hypercapnia and isocapnic hypoxia. J. Appl. Physiol. 1979; 46:1096-1102. [PubMed: 468630] 


\section{Highlights}

- HF-SCS results in activation of the diaphragm at physiological firing frequencies

- HF-SCS results in the generation of large inspired volume in the rat model

- Continuous HF-SCS pacing can be maintained without evidence of system fatigue 


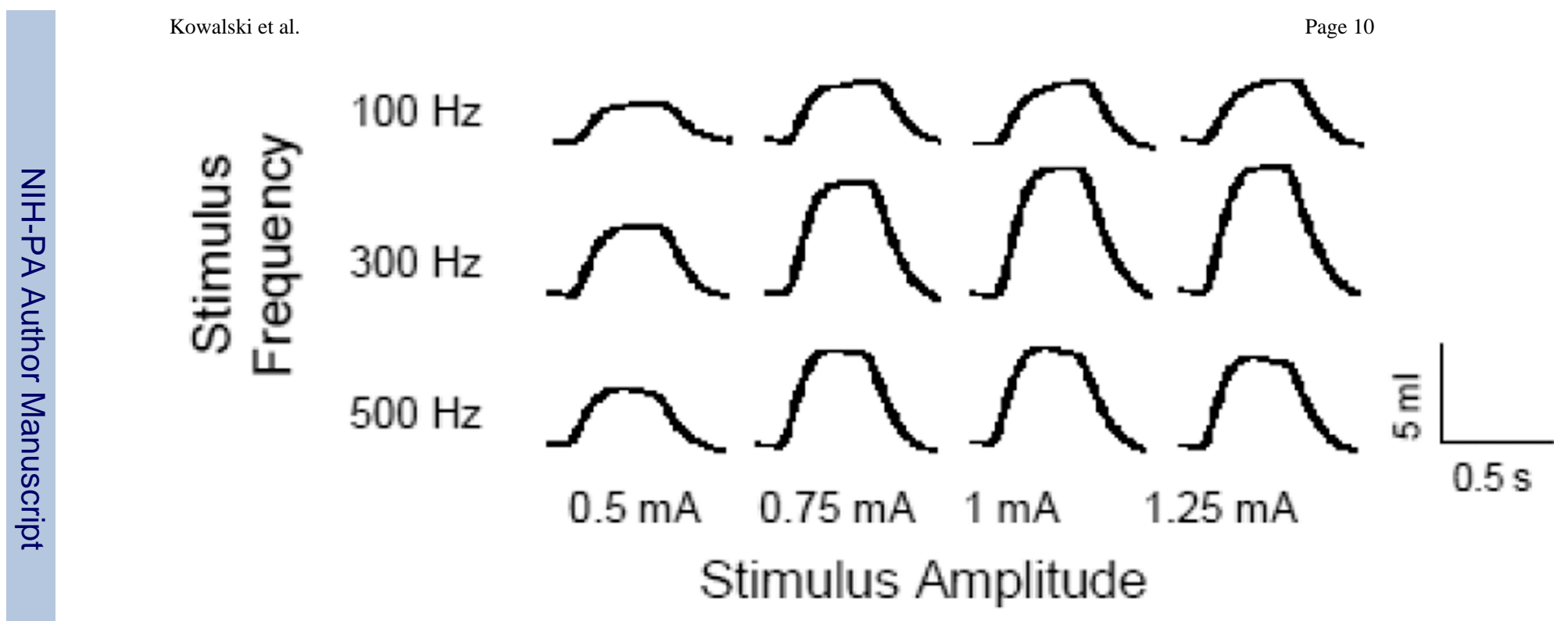

Figure 1.

Comparison of different stimulus amplitudes and stimulus frequencies on inspired volume generation during HF-SCS in a representative animal. 


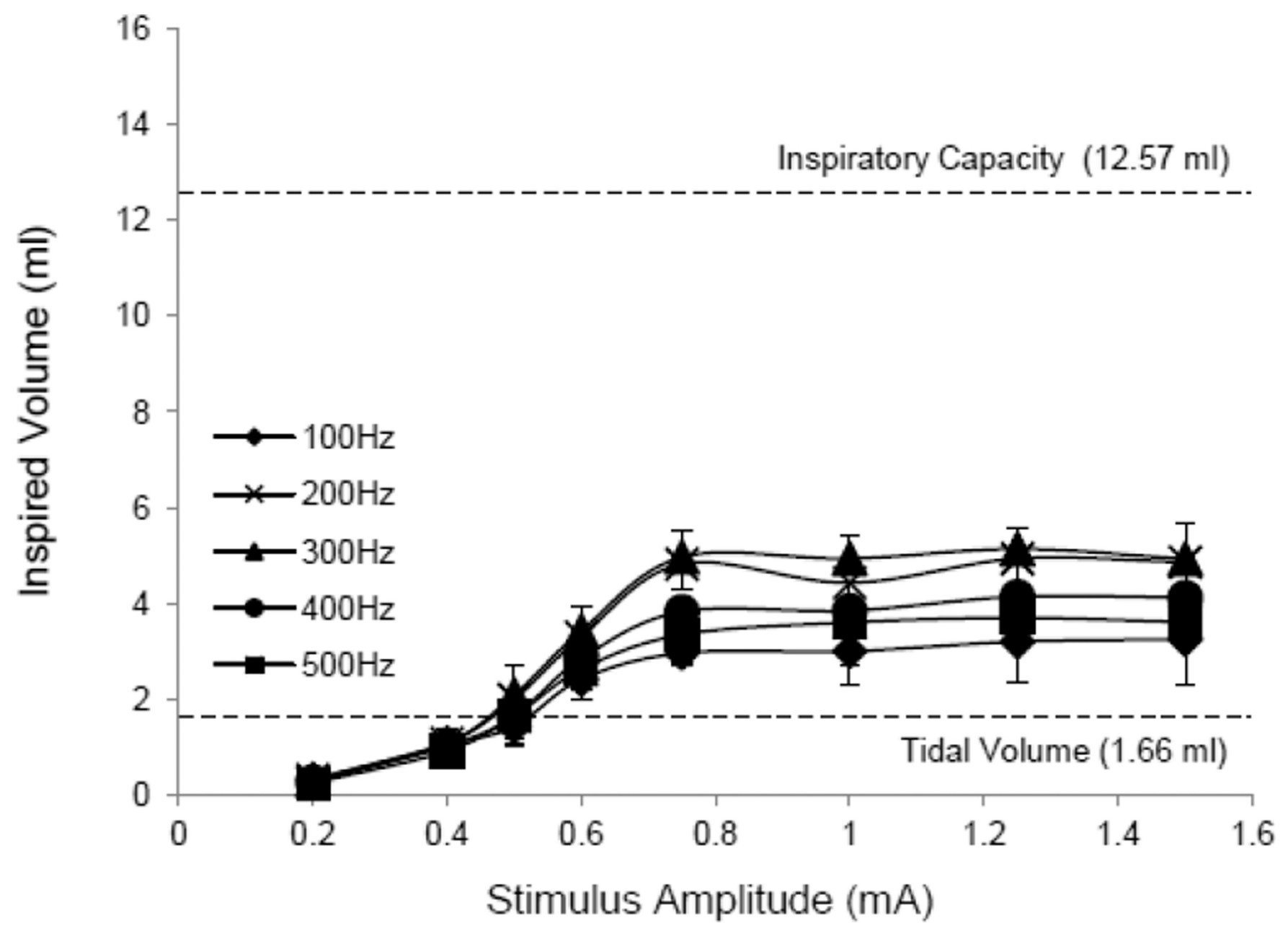

Figure 2.

Relationship between stimulus amplitude and inspired volume generation at different stimulus frequencies. With increasing stimulus frequencies between 100 and $300 \mathrm{~Hz}$, there were increases in the magnitude of inspired volume generation. The 200 and $300 \mathrm{~Hz}$ curves were not statistically different. Stimulation at higher frequencies (400 and $500 \mathrm{~Hz}$ ), however, resulted in decrements in inspired volume generation which were significantly lower than that achieved with $300 \mathrm{~Hz}$ but greater than that achieved with $100 \mathrm{~Hz}$. See Results for further description. 


\section{Spontaneous Breathing Control}

\section{High Frequency SCS After $\mathrm{C} 1$ section}
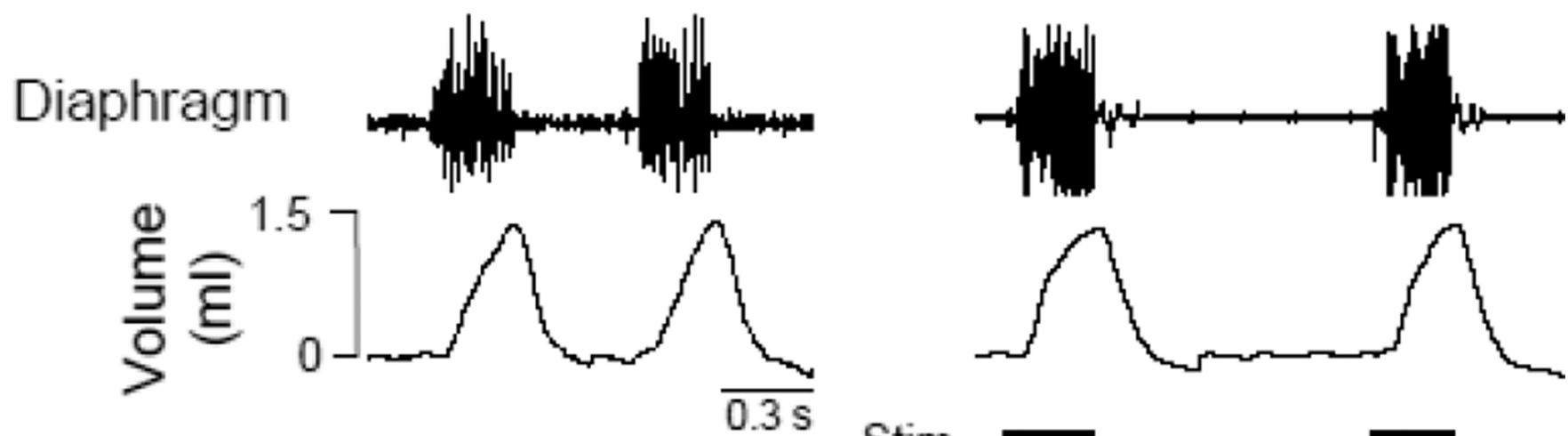

Figure 3.

Multiunit EMGs of the diaphragm during spontaneous breathing (left panel) and during HFSCS (right panel). Stimulus parameters during HF-SCS were adjusted to approximate inspired volumes during spontaneous breathing. See Results for further explanation. 


\section{Spontaneous Breathing Control}

\section{High Frequency SCS After $\mathrm{C} 1$ section}

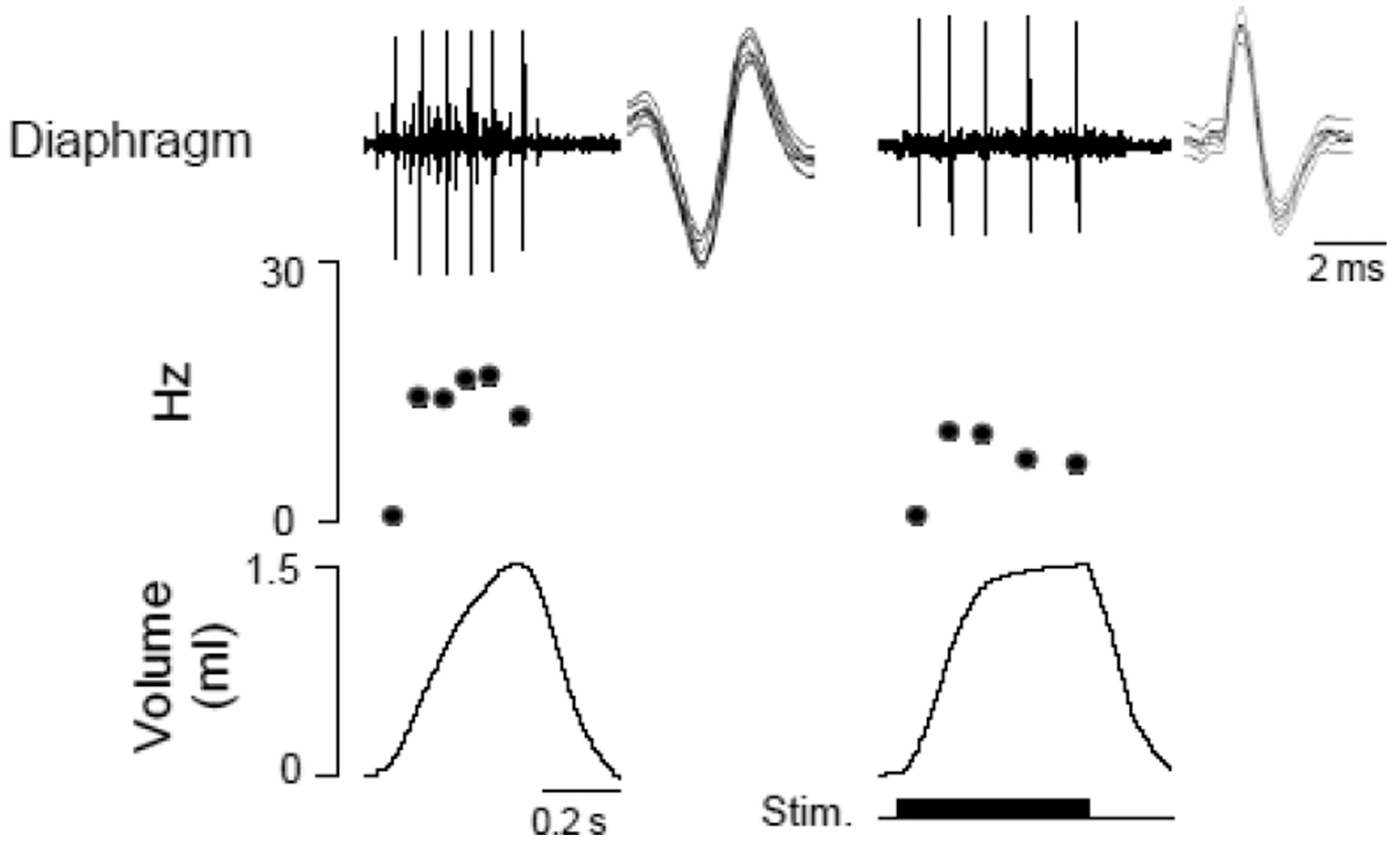

Figure 4.

Single motor unit recordings from the diaphragm muscle during spontaneous breathing (left panel) and HF-SCS (right panel) in one animal. From top to bottom, tracings represent single motor unit recordings, instantaneous SMU firing frequencies and inspired volume. Recordings were obtained during HF-SCS with adjustment of stimulus amplitude to match the inspired volume observed during spontaneous breathing. The action potentials from each motor unit are superimposed at the right of each tracing. See Results for further explanation. 


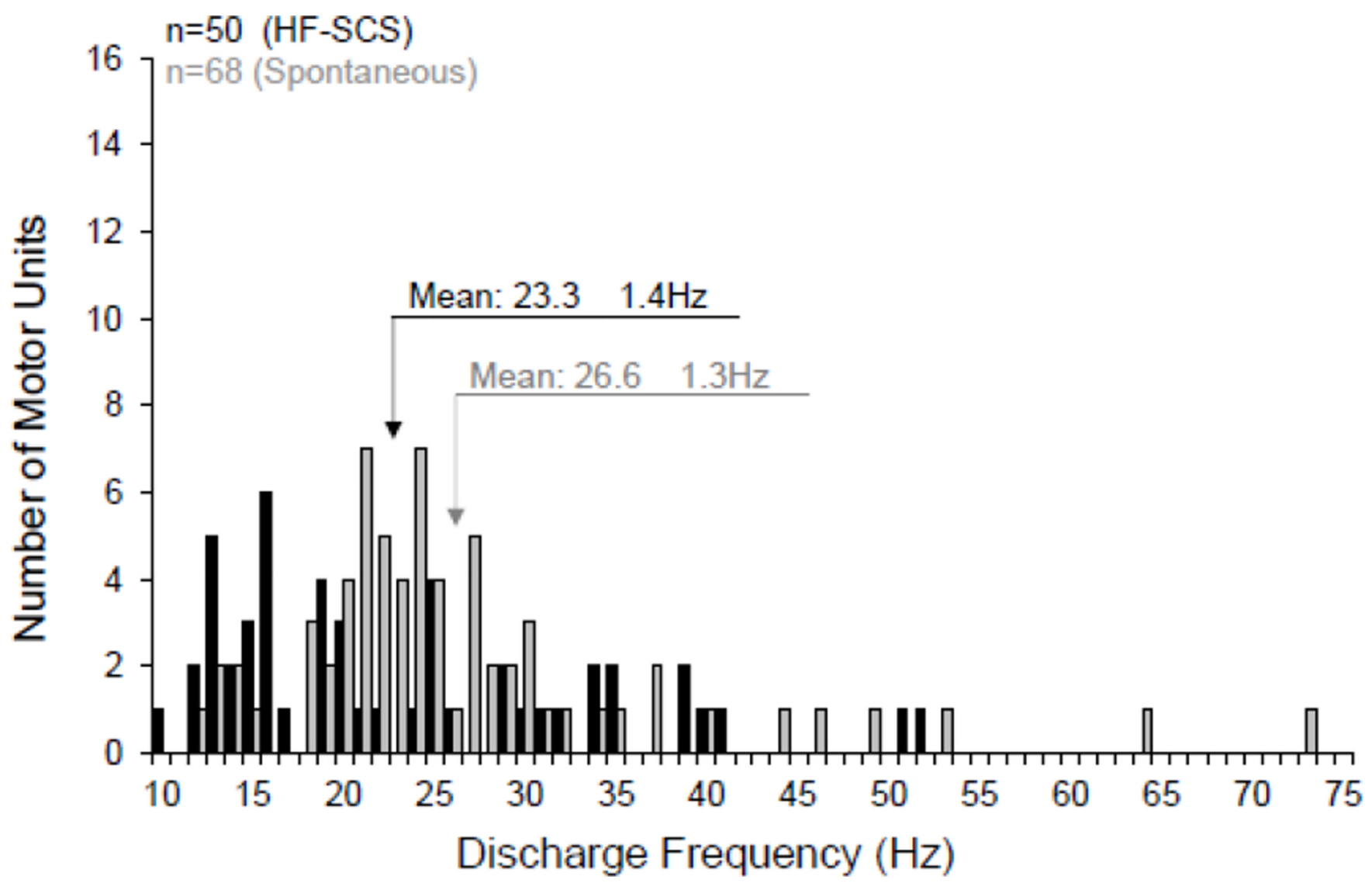

Figure 5.

Firing frequency of each motor unit recorded from the diaphragm during spontaneous breathing (gray bars) and HF-SCS (black bars). Bin width: 1.0 Hz. See Results for further explanation. 

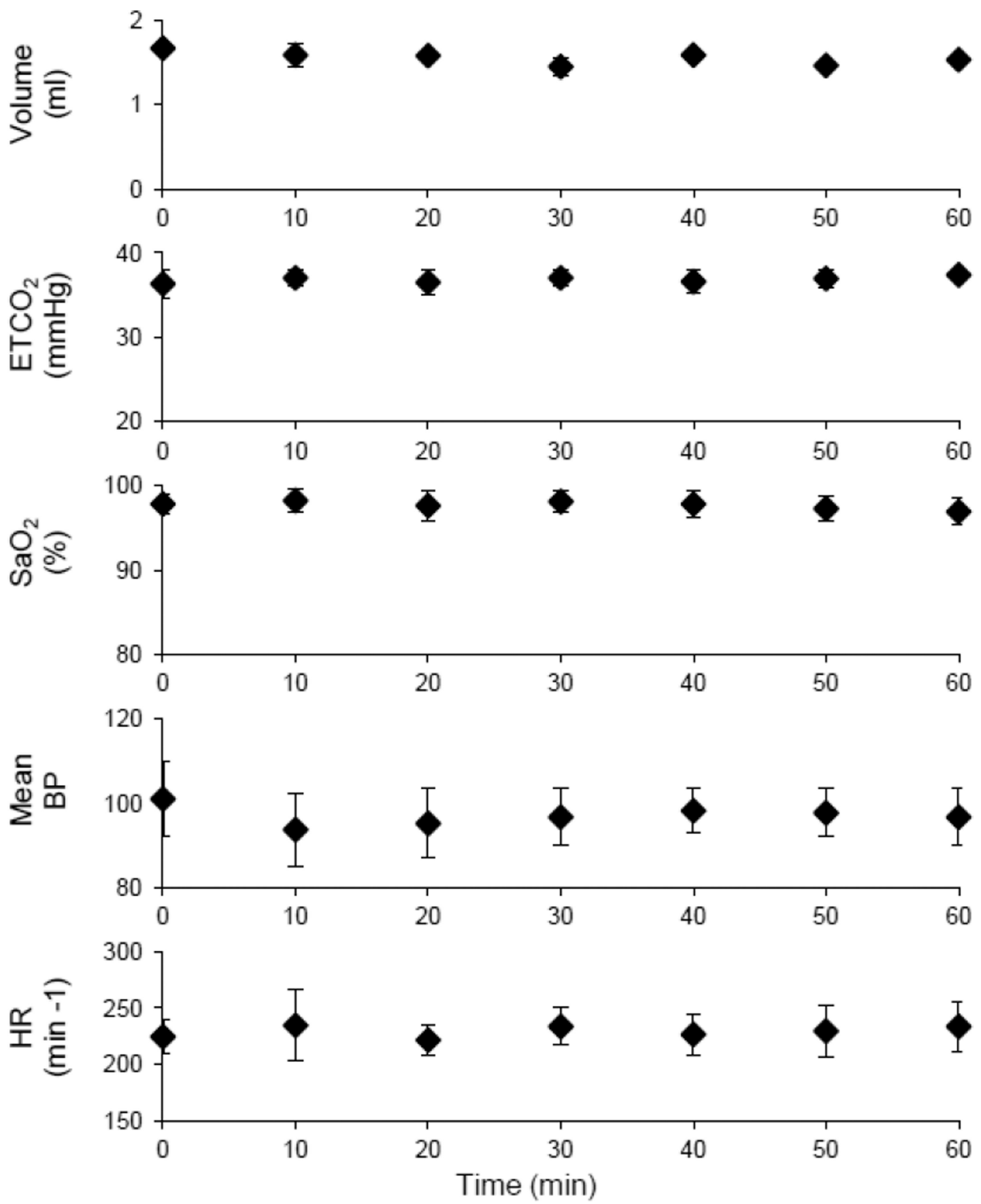

Figure 6.

Inspired volume, end-tidal $\mathrm{PCO}_{2}$, oxygen saturation $\left(\mathrm{SaO}_{2}\right)$, mean blood pressure $(\mathrm{BP})$ and heart rate (HR) plotted every 10 min during inspiratory muscle pacing with HF-SCS. Results are mean $\pm \mathrm{SE}(\mathrm{n}=3)$. Inspired volume remained constant throughout the period of stimulation. End-tidal $\mathrm{PCO}_{2}$ and oxygen saturation were also maintained between 35 and 40 mmHg and 96 and 99\%, respectively. Mean blood pressure and heart rate also remained stable. See Table 1. 


\section{Table 1}

Ventilatory parameters during $60 \mathrm{~min}$ of continuous HF-SCS pacing (Stimulus parameters: $300 \mathrm{~Hz}, 0.5 \mathrm{~mA}$, $0.2 \mathrm{~ms}$ pulse width)

\begin{tabular}{ccc}
\hline $\begin{array}{c}\text { Time } \\
(\mathbf{m i n})\end{array}$ & $\begin{array}{c}\mathbf{E T C O}_{2} \\
(\mathbf{m m H g})\end{array}$ & $\begin{array}{c}\mathrm{SaO}_{2} \\
(\%)\end{array}$ \\
\hline 0 & $36.3 \pm 1.7$ & $97.9 \pm 1.2$ \\
10 & $37.0 \pm 1.0$ & $98.3 \pm 1.5$ \\
20 & $36.5 \pm 1.5$ & $97.7 \pm 1.9$ \\
30 & $37.0 \pm 1.0$ & $98.2 \pm 1.4$ \\
40 & $36.6 \pm 1.4$ & $97.9 \pm 1.7$ \\
50 & $36.9 \pm 1.1$ & $97.3 \pm 1.5$ \\
60 & $37.4 \pm 0.6$ & $97.0 \pm 1.6$ \\
\hline
\end{tabular}

